\title{
Isolation, Identification and Characterization of Enteric Bacteria from Post Weaning Diarrheic Pigs and their Resistance to Multiple Antibiotics
}

\author{
Dinesh Mittal $^{1}$, Kushal Grakh ${ }^{1}$, Anand Prakash ${ }^{1}$, Pallavi Moudgil ${ }^{1}$, \\ Bhanita Devi ${ }^{2}$ and Vijay Jadhav ${ }^{1}$ \\ ${ }^{1}$ Department of Veterinary Public Health and Epidemiology, ${ }^{2}$ Department of Animal \\ Biotechnology, LUVAS, Hisar, Haryana, India \\ *Corresponding author
}

\section{Keywords}

Antibiotic resistance,

E. coli, Post weaning

diarrhea, Swine

production,

VITEK®2

\section{Article Info}

Accepted:

17 November 2018

Available Online:

10 December 2018
A B S T R A C T

In the present study, 25 diarrheic faecal samples/ rectal swabs collected from post weaning pigs were examined by cultural method for the presence of Enterobacteriaceae members which resulted in isolation of 18 lactose fermenter and two non-lactose fermenter bacterial colonies. The recovered colonies were subjected to VITEK ${ }^{\circledR} 2$ Compact System for the identification of bacteria. Out of 18 lactose fermenter bacterial colonies, 15 were identified as Escherichia coli, two as Enterobacter asburiae, and one as Klebsiella pneumoniae. Both the lactose non-fermenter bacterial colonies were identified as Pseudomonas aeruginosa. In-vitro antibiotic sensitivity pattern of E. coli isolates showed $100 \%$ sensitivity to chloramphenicol, gentamicin and ampicillin/sulbactam whereas $60 \%$ isolates were found to be resistant to co-trimoxazole and 53\% isolates to amoxicillin clavulanate and cefotaxime. All E. coli isolates showed resistance to more than four antibiotics, four isolates showed resistance to six antibiotics and two isolates showed resistance to more than seven antibiotics. Isolates of $K$. pneumoniae, Enterobacter asburiae and $P$. aeruginosa found to be sensitive to most of the antibiotics tested; whereas these isolates showed resistance to amoxycillin/clavulanate and co-trimoxazole. This study helps in assessment of bacterial resistance of enteric bacteria recovered from diarrheic pigs.

\section{Introduction}

Diarrhea in pigs is one of the major problems worldwide leading to huge economic losses to swine industry. Neonatal and post-weaning diarrhea (PWD) is responsible for high morbidity and mortality where mortality may reach up to 20-30\% during acute outbreaks of PWD (Amezcua et al., 2002). Many viral, bacterial, and parasitic agents are involved in the cause of neonatal diarrhea such as transmissible gastroenteritis (TGE) virus, swine enteric coronavirus disease (SECD) including porcine epidemic disease virus (PEDV) and swine delta corona virus (SDCV), rotavirus, E. coli, Salmonella, Clostridium perfringens type C, Strongyloides ransomi and Coccidia. PWD, however, is a multifactorial as it involves interaction between sow, piglet, environment, ETEC bacteria and managemental practices (Hong et al., 2006). Though exact cause has not yet 
been identified for PWD in pigs (Jensen et al., 2006), E. coli is considered as main bacterial cause for PWD which can cause infection alone or in combination with rotavirus. Neonatal and post weaning diarrhea or enteric collibacillosis alone may result in heavy economic losses due to decreased weight gain, cost of treatment, vaccination, use of feed supplements and mortality (Fairbrother et al., 2012). In India, the most common cause of morbidity and mortality in piglets is diarrhea (Sinha et al., 2018) and E. coli is primarily associated with these diarrheic pigs as reported previously (Begum et al., 2014; Sikdar, 1991; Begum et al., 2014; Regon et al., 2014; Borah, 1994; Shome et al., 2005). To control PWD, antimicrobials are widely used in piggeries for prophylactic and therapeutic purposes. The most commonly used antimicrobials to treat affected piglets are amoxycillin/clavulanate, trimethoprim/ sulphonamide, enrofloxacin, colistin, apramycin, ceftiofur, neomycin and gentamicin (Fairbrother et al., 2012). Frequent and long-term use of a particular antibiotic in a specific region may results in the development of resistance in the bacteria to the particular antibiotic (Moon et al., 2007). The multiple antibiotic resistance strains of $E$. coli emerged as formidable threat to piggery industry (Mathew et al., 1999) and may spill over to other livestock as well as human population (Osterberg et al., 2016; Valiakos et al., 2016). Therefore, there is a constant need to update the antibiotic sensitivity profile of $E$. coli isolated from piggeries, particularly from diarrheic pigs, to help in selection of effective antibiotics for therapeutic purposes, to reduce treatment cost, and to combat antibiotic resistance.

In the present study, Enterobacteriaceae members were isolated from the post weaned diarrheic pigs, characterized using VITEK $^{\circledR} 2$ system and subjected to antibiotic susceptibility testing to assess the sensitivity/resistance of isolates.

\section{Materials and Methods}

\section{Collection of samples and processing}

A total of 25 fecal samples/rectal swabs were collected from piglets from pig farms from Uttar Pradesh and Haryana, with clinical signs of diarrhea, dehydration and decreased weight gain. The samples were collected with sterile swabs under aseptic conditions and transported to the laboratory immediately under cold conditions. These samples were then diluted using $0.9 \%$ normal saline solution and processed for the isolation of bacteria.

\section{Isolation and characterization of bacteria}

Normal saline diluted fecal samples were streaked on MacConkey Lactose Agar (MLA) for the isolation of members of Enterobacteriaceae family. The plates were then incubated at $37^{\circ} \mathrm{C}$ for 24 hours under aerobic conditions.

The isolated colonies were subjected to Gram's staining. The lactose fermenter and lactose non-fermenter colonies were further subcultured to obtain pure colonies. The purified lactose fermenter colonies were then streaked on eosin methylene blue (EMB) agar and lactose non-fermenter colonies on brilliant green agar (BGA) for further characterization.

\section{Microbial identification using VITEK®2 Compact System}

Gram negative (GN) reagent cards having 64 wells each containing an individual test substrate were used. A sufficient number of colonies of pure culture of microorganisms were suspended in $3.0 \mathrm{ml}$ of sterile saline (aqueous $0.50 \% \mathrm{NaCl}, \mathrm{pH} 4.5$ to 7.0 ) in a $12 \times 75 \mathrm{~mm}$ (polystyrene test tube. The turbidity was adjusted accordingly (for Gram negative bacteria, McFarland turbidity range should be 0.50-0.63) and measured using a 
turbidity meter, DensiCheck ${ }^{\mathrm{TM}}$ and the cassettes with tubes and reagent cards were loaded in VITEK ${ }^{\circledR} 2$ Compact system and the results were interpreted as per instructions of manufacturer (BioMérieux, Inc. USA).

\section{In- vitro antibiotic sensitivity}

In vitro single disc diffusion technique (Bauer et al.,1966) was employed using antibiotic discs viz., amoxycillin/clavulanate (20/10 $\mathrm{mcg})$, ampicillin/sulbactam (10/10 mcg), cefoperazone $(75 \mathrm{mcg})$, cefotaxime $(30 \mathrm{mcg})$, chloramphenicol (30 $\mathrm{mcg})$, ciprofloxacin (5 $\mathrm{mcg})$, cotrimoxazole $(25 \mathrm{mcg})$, gentamicin (10 $\mathrm{mcg}$ ), kanamycin (30 mcg), levofloxacin (5 $\mathrm{mcg})$, moxifloxacin $(5 \mathrm{mcg})$ and tetracycline $(30 \mathrm{mcg})$ for assessment of antibiotic susceptibility testing. The plates were incubated at $37^{\circ} \mathrm{C}$ for 24 hours under aerobic conditions. The results were then interpreted on the basis of inhibition zone as per the manufacturer's recommendations (Hi-Media, India).

\section{Results and Discussion}

Out of 25 samples processed, 18 recovered isolates exhibited lactose fermenter pink colonies and two recovered isolates exhibited non-fermenter pale colonies on MLA agar (Fig. 1A and B). Of 18 lactose fermenters, three had mucoid appearance and rest had flat colonies with entire margins. All 18 isolates were found to be Gram negative rods under oil immersion of microscope upon Gram's staining (Fig. 1C and D; Table 1). These 18 lactose fermenter isolates when streaked on EMB agar showed variable results. Twelve isolates showed green metallic sheen (Fig. $1 \mathrm{E})$, five isolates showed flat, dark colored colonies and one isolate showed dark colored, mucoid colonies with no metallic sheen. The two non-lactose fermenter colonies on BGA showed pinkish, round colonies (Fig. 1F).
The lactose fermenter isolates with and without green metallic sheen on EMB agar were confirmed as E. coli by $\operatorname{VITEK}^{\circledR} 2$ Compact System. Of confirmed 15 bacterial isolates to be of $E$. coli, only 12 isolates showed green metallic sheen on EMB agar and three did not. The probable reason behind lack of green metallic sheen may be due to transition of $E$. coli from smooth to rough variant where bacteria lose some of its morphology and other characters. The results of the study indicated that frequency of isolation of $E$. coli from fecal samples was $60 \%$. Primarily work on porcine E. coli had been carried out in north eastern state of India where pigs are raised in larger number. Isolation frequency varied in north eastern state of India ranging from 15\% (Begum et al., 2014), 51.95\% (Sikdar, 1991), 59.3\% (Begum et al., 2014), 60\% (Regon et al., 2014), 86.11 $\%$ (Borah, 1994), and 90\% (Shome et al., 2005). Similarly, pigs are raised extensively in Asian countries such as China, Taiwan, Japan and Vietnam where the outbreaks of PWD are quite common (Lee, 2015). The present study is quite close to results of Japan where E. coli was isolated from $58.1 \%$ of weaned piglets (Katsuda et al., 2006). E. coli from weaned piglets were isolated at a lower frequency at $17.6 \%$ and $22.4 \%$ respectively in Germany and Canada (Morin et al., 1983; Wieler et al., 2001). The variation in $E$. coli isolation rate could be explained by geographical location, type of antibiotic usages, age of the animals, concurrent infections and no. of samples tested. Two of the bacterial isolates were confirmed as Enterobacter asburiae and one isolate as Klebsiella pneumoniae. There are no reports of Enterobacter asburiae causing diarrheic symptoms in pigs. Klebsiella pneumoniae subspp. pneumoniae is a commensal as well as the cause of septicemia in pigs (Bidewell et al., 2018). Though Klebsiella pneumoniae have not been directly reported from diarrheic pigs but have been able to experimentally induce diarrhea in pigs 
(Wilcock, 1978). The two isolates which were non-lactose fermenter on MLA and gave pinkish red colonies on BGA were confirmed as Pseudomonas aeruginosa using VITEK $^{\circledR} 2$ System. On Mueller Hinton agar plates, the organism also produced green pigment. There are no published reports of $P$. aeruginosa being involved in porcine diarrhea. So, mere isolation of any bacteria could not be attributed to diarrhea. Even though normal and apparently healthy pigs also yield $E$. coli in their feces but the presence of $E$. coli in cases of diarrheic cases could not be understated as it is a part of multifactorial etiology of PWD. However the pathogenic nature of the isolates needs to be established using molecular characterization of various virulence gens such as toxin, bundle forming pilli etc. Overall, in the present study, 20 (80\%) bacterial isolates were recovered from 25 diarrheic pigs, with $E$. coli in majority of cases $(60 \%)$.

In the present study, out of 12 antibiotics assessed, 15 E. coli isolates showed multiple drug resistance to nine antibiotics (Table 2 and Fig. 2). There was more than $50 \%$ resistance to five antibiotics viz. co-trimoxazole, amoxicillin clavulanate, moxifloxacin, cefoperazone and cefotaxime with maximum resistance of $60 \%$ was observed for cotrimoxazole followed by amoxicillin clavulanate and cefotaxime (53.3\%), moxifloxacin and cefoperazone (40\%), tetracycline (33.3\%), ciprofloxacin (23.3\%), levofloxacin $(20 \%)$ and kanamycin (13.3\%). The antibiotic susceptibility of two isolates of $P$. aeruginosa, 2 isolates of E. asburiae and the only isolate of $K$. pneumoniae subspp pneumoniae was also determined.

Table.1 Prevalence of $E$. coli and other bacteria isolated from 25 diarrheic faecal samples of pigs

\section{Bacteria}

E. coli

E. asburiae

P. aeruginosa

K. pneumoniae subspp. pneumoniae

Total

\section{Number of bacterial} isolate

15

2

2

1

20

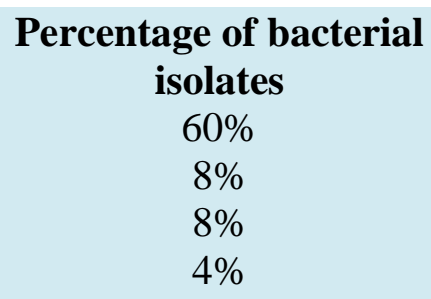

$80 \%$

Table.2 Antibiotic susceptibility pattern of $E$. coli isolated from diarrheic pigs faecal samples

\begin{tabular}{cccc}
\hline Antibiotics & Sensitive (\%) & Moderate sensitive (\%) & Resistant (\%) \\
Ampicillin/Sulbactam & $15(100 \%)$ & - & - \\
Amoxicillin clavulanate & $1(6.66 \%)$ & $6(40 \%)$ & $8(53.33 \%)$ \\
Cefoperazone & $4(26.66 \%)$ & $5(33.33 \%)$ & $6(40 \%)$ \\
Cefotaxime & $3(20 \%)$ & $4(26.66 \%)$ & $8(53.33 \%)$ \\
Chloramphenicol & $15(100 \%)$ & - & - \\
Ciprofloxacin & $9(60 \%)$ & $2(13.33 \%)$ & $4(26.66 \%)$ \\
Co-trimoxazole & $5(33.33 \%)$ & $1(6.66 \%)$ & $9(60 \%)$ \\
Gentamicin & $15(100 \%)$ & - & - \\
Kanamycin & $9(60 \%)$ & $4(26.66 \%)$ & $2(13.33 \%)$ \\
Levofloxacin & $10(66.66 \%)$ & $2(13.33 \%)$ & $3(20 \%)$ \\
Moxifloxacin & $8(53.33 \%)$ & $1(6.66 \%)$ & $6(40 \%)$ \\
Tetracycline & $7(46.66 \%)$ & $3(20 \%)$ & $5(33.33 \%)$
\end{tabular}


Fig.1 Isolation and characterization of bacterial isolates (A) Lactose fermenter colonies on MLA (B) Lactose non-fermenter colonies on MLA (C) Gram's staining showing gram negative E. coli cells (D) Gram's staining showing gram negative lactose non-fermenter Pseudomonas aeruginosa (E) E. coli colonies with green metallic sheen on EMB agar (F) Pink colonies of $P$. aeruginosa on BGA
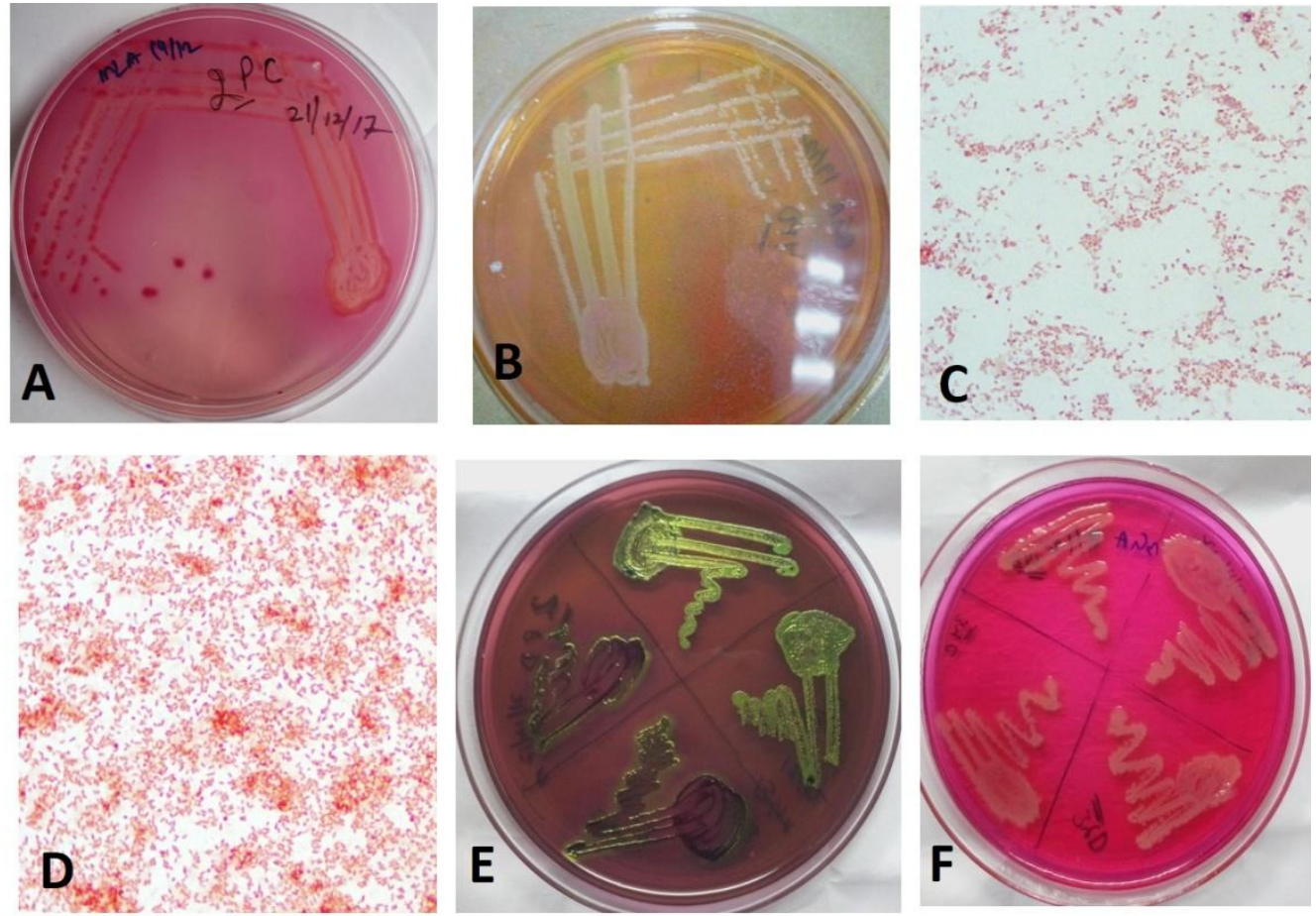

Fig.2 Antibiotic susceptibility testing using disc diffusion method (A) E. coli (B) P. aeruginosa. The antibiotics used were amoxicillin clavulanate $(20 / 10 \mathrm{mcg})$, ampicillin/sulbactam (10/10 $\mathrm{mcg}$ ), cefoperazone (75 mcg), cefotaxime (30 mcg), chloramphenicol (30 mcg), ciprofloxacin (5 $\mathrm{mcg})$, cotrimoxazole $(25 \mathrm{mcg})$, gentamicin $(10 \mathrm{mcg})$, kanamycin $(30 \mathrm{mcg})$, levofloxacin $(5 \mathrm{mcg})$, moxifloxacin $(5 \mathrm{mcg})$ and tetracycline $(30 \mathrm{mcg})$

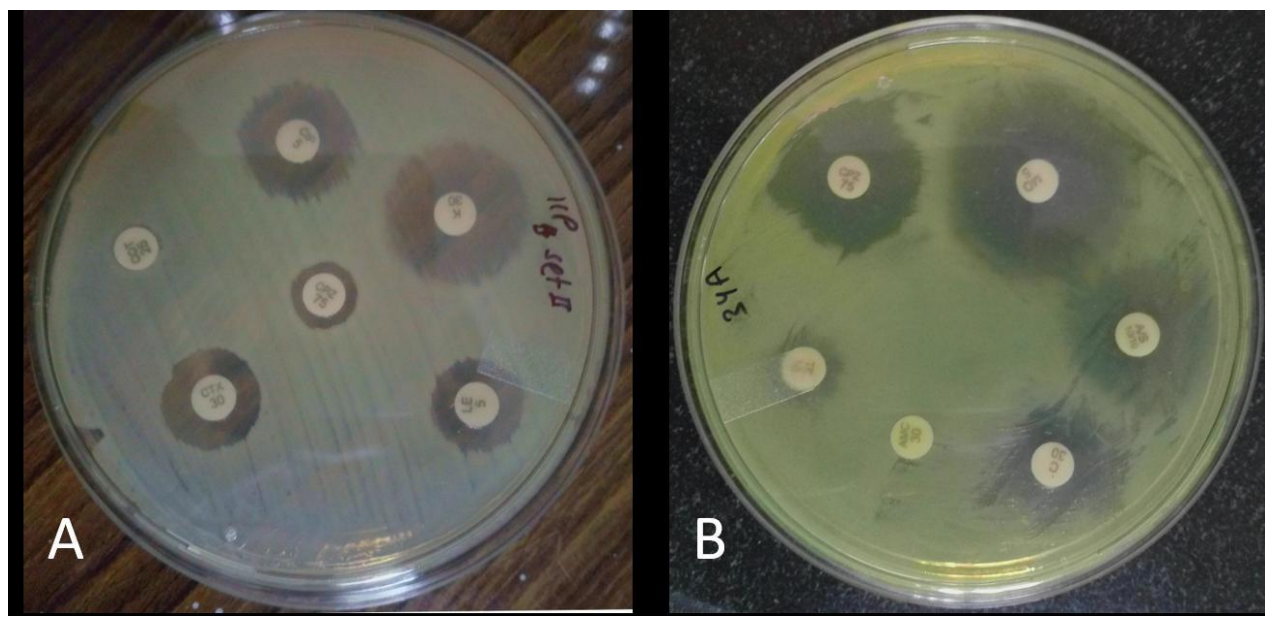


The results showed that all five isolates were $100 \%$ sensitive to gentamicin, ampicillin/sulbactam and cefoperazone. The $P$. aeruginosa isolates were $100 \%$ sensitive to moxifloxacin, ciprofloxacin, levofloxacin whereas E. asburiae isolates were $100 \%$ sensitive to ciprofloxacin, cefotaxime, cefoperazone, gentamicin and ampicillin/ sulbactam. The only isolate of $K$. pneumoniae pneumoniae was found to be sensitive to chloramphenicol, gentamicin, ampicillin/ sulbactam and levofloxacin. All three $(P$. aeruginosa, E. asburiae and $K$. pneumoniae pneumonia) bacteria were completely resistant to amoxicillin clavulanate and cotrimoxazole. The presence of antibiotic resistance genes primarily among E. coli isolates explains why some diarrheic cases were refractory to antibiotic therapies.

Escherichia coli is a Gram-negative bacteria of family Enterobacteriaceae and the causal and associated agent of various diseases of pigs, including neonatal diarrhea and PWD, which are responsible for death in suckling and weaned pigs, respectively worldwide (Fairbrother et al., 2012). Depending upon the severity of the disease, the cost of PWD was estimated to vary from $€ 40$ to $€ 314$ per sow (Sjölund et al., 2014), which is equivalent to INR 3200-25250 per sow. This is in addition to the heavy losses in piglets due to decreased weight gain, cost of treatment, vaccination, use of feed supplements and mortality (Fairbrother et al., 2012). In the present study, the pig owners have informed us that they do not use antibiotics as feed additives and hence the presence of antibiotic resistant strains of bacteria in piglets might be due to nonprudent use of antibiotics by unqualified people in that area. During the outbreaks of PWD, quick actions are required and hence the antibiotic treatment is prescribed well before the results of sensitivity testing. Therefore, it is the responsibility of practitioner to choose effective antibiotics (Luppi, 2017).
To maintain a profitable porcine industry, it is very essential to diagnose the etiological agent of PWD. In case of bacterial involvement, the assessment of antibiotic susceptibility pattern for timely treatment, to reduce the unnecessary cost and development of antibiotic resistance is must. As the organisms are acquiring resistance against commonly used antibiotics, determination of antimicrobial sensitivity test becomes necessary, so that non-prudent use of antibiotics and development of resistance can be prevented. This would also help in preventing the spillover of the antibiotic resistant strains to other livestock and human population.

\section{Acknowledgements}

The authors are thankful to Prof. \& Head, Department of VPHE, LUVAS, Hisar for providing necessary facilities to carry out the work. Also authors want to extend their thanks to Dr. N.K. Mahajan and Dr. Naresh Jindal for their valuable suggestions, constant support and encouragement.

\section{References}

Amezcua, R., Friendship, R. M., Dewey, C. E., Gyles, C. and Fairbrother, J. M. 2002. Presentation of postweaning Escherichia coli diarrhea in southern Ontario, prevalence of hemolytic E. coli serogroups involved, and their antimicrobial resistance patterns. Can. J. Vet. Res., 66 (2): 73.

Begum, J., Dutta, T. K., Chandra, R., Choudhary, P. R., Varte, Z. and Bitew, M. 2014. Molecular and phenotypic characterization of shigatoxigenic Escherichia coli (STEC) and enteropathogenic E. coli (EPEC) from piglets and infants associated with diarrhoea in Mizoram, India. Afr. $J$. Biotechnol., 13 (12): 1452-1461. 
Begum, S., Hazarika, G. C. and Rajkhowa, S. 2014. Prevalence of Escherichia coli from pigs and cattle. J. Anim. Health Prod., 2 (3): 38-39.

Bhushan, B. 2018. Comparative jejunal expression of MUC 13 in Indian native pigs differentially adhesive to diarrhoeagenic E. coli. J. Appl. Anim. Res., 46 (1): 107-111.

Bidewell, C. A., Williamson, S. M., Rogers, J., Tang, Y., Ellis, R. J., Petrovska, L. and AbuOun, M. 2018. Emergence of Klebsiella pneumoniae subspecies pneumoniae as a cause of septicaemia in pigs in England. PloS One., 13 (2): e0191958.

Borah, P. 1994. An epidemiological study on gastroenteritis in pigs. Ph.D. thesis, Assam Agric. Univ., Guwahati, India.

Do, T. N., Cu, P. H., Nguyen, H. X., Au, T. $\mathrm{X} ., \mathrm{Vu}, \mathrm{Q} . \quad \mathrm{N}$. , Driesen, S. T., Townsend, K. M., Chin, J. J. C and Trott, D. J., 2006. Pathotypes and serogroups of enterotoxigenic Escherichia coli isolated from preweaning pigs in North Vietnam. Med. Microbiol., 55: 93 - 99.

Fairbrother, J.M. and Gyles, C. 2012 Colibacillosis. In: Diseases of Swine. Zimmerman, J.J., Karriker, L.A., Ramirez, A., Schwartz, K.J., Stevenson, G.W. 10th Ed., John Wiley \& Sons, Hoboken, New Jersey, United States. pp. 723-47.

Hong, T. T. T., Linh, N. Q., Ogle, B. and Lindberg, J. E. 2006. Survey on the prevalence of diarrhoea in pre-weaning piglets and on feeding systems as contributing risk factors in smallholdings in Central Vietnam. Trop. Anim. Health Prod., 38 (5): 397- 405.

Jensen, G. M., Frydendahl, K., Svendsen, O., Jørgensen, C. B., Cirera, S., Fredholm, M. and Møller, K. 2006. Experimental infection with Escherichia coli O149: F4ac in weaned piglets. Vet. Microbiol.,
115 (1-3): 243-249.

Katsuda, K., Kohmoto, M., Kawashima, K. and Tsunemitsu, H. 2006. Frequency of enteropathogen detection in suckling and weaned pigs with diarrhea in Japan. J.Vet. Diagn. Investig., 18 (4): 350-354.

Luppi, A. 2017. Swine enteric colibacillosis: diagnosis, therapy and antimicrobial resistance. Porcine Health Manag., 3 (1): 16.

Mathew, A. G., Saxton, A. M., Upchurch, W. G. and Chattin, S. E. 1999. Multiple Antibiotic Resistance Patterns of Escherichia coli Isolates from Swine Farms. Appl. Environ. Microbiol., 65 (6): 2770-2772.

Moon, J. S., Lee, A. R., Kang, H. M., Lee, E. S., Kim, M. N., Paik, Y. H. and Koo, H. C. 2007. Phenotypic and genetic antibiogram of methicillin-resistant staphylococci isolated from bovine mastitis in Korea. J. Dairy Sci., 90 (3): 1176-1185.

Morin, M., Turgeon, D., Jolette, J., Robinson, Y., Phaneuf, J. B., Sauvageau, R. and Lariviere, S. 1983. Neonatal diarrhea of pigs in Quebec: infectious causes of significant outbreaks. Can. J. Comp. Med., 47 (1): 11.

Österberg, J., Wingstrand, A., Jensen, A. N., Kerouanton, A., Cibin, V., Barco, L. and Bengtsson, B. 2016. Antibiotic resistance in Escherichia coli from pigs in organic and conventional farming in four European countries. PloS One, 11 (6): e0157049.

Regon, M., Pathak, D. C., Tamuli, S. M. and Baruah, G. K. 2014. Serotyping of Escherichia coli isolated from piglet diarrhea. Vet. World. 7 (8): 614-616.

Rhouma, M., Fairbrother, J. M., Beaudry, F. and Letellier, A. 2017. Post weaning diarrhea in pigs: risk factors and noncolistin based control strategies. Acta Vet. Scan., 59 (1): Shome R., Shome, B.R., Rahman, M., Kumar, A., 
Murugkar, H.V., Rahman, H. and

Bujarbaruah, K. 2005. Plasmid diversity in Escherichia coli strains isolated from piglet diarrhoea. Indian. J. Anim. Sci. 75:196-198.

Sikdar, D. 1991. Escherichia coli in piglet diarrhea: Enterotoxigenicity and drug sensitivity. M.V.Sc. Thesis, Assam Agric. Univ., Guwahati, India.

Sinha, R., Sahoo, N. R., Kumar, P., Qureshi, S., Kumar, A., Ravikumar, G. V. P. P. S. and Lee, C. (2015). Porcine epidemic diarrhea virus: an emerging and reemerging epizootic swine virus. Virol. J., 12 (1): 193.

Sjolund, M. 2014. Financial impact on pig production III: Gastrointestinal disorders. In: Proceedings of the 6th European Symposium of Porcine Health
Management, Sorrento, Italy, 189.

Valiakos, G., Vontas, A., Tsokana, C.N., Giannakopoulos, A., Chatzopoulos, D. and Billinis, C. 2016. Resistance in Escherichia coli Strains Isolated from Pig Faecal Samples and Pig farm Workers, Am. J. Anim. Vet. Sci., 11 (4): 142-144.

Wieler, L. H., Ilieff, A., Herbst, W., Bauer, C., Vieler, E., Bauerfeind, R. and Zahner, H. 2001. Prevalence of enteropathogens in suckling and weaned piglets with diarrhoea in southern Germany. Zoonoses Public Health, 48 (2): 151-159.

Wilcock, B. P. 1979. Experimental Klebsiella and Salmonella infection in neonatal swine. Can. J. Comp. Med., 43 (2): 200206.

\section{How to cite this article:}

Dinesh Mittal, Kushal Grakh, Anand Prakash, Pallavi Moudgil, Bhanita Devi and Vijay Jadhav. 2018. Isolation, Identification and Characterization of Enteric Bacteria from Post Weaning Diarrheic Pigs and their Resistance to Multiple Antibiotics. Int.J.Curr.Microbiol.App.Sci. 7(12): 2377-2384. doi: https://doi.org/10.20546/ijcmas.2018.712.269 